\title{
Neuroendocrine tumors of the thymus and mediastinum
}

\author{
Hanibal Bohnenberger ${ }^{1}$, Helen Dinter ${ }^{1}$, Alexander König ${ }^{2}$, Philipp Ströbel ${ }^{1}$ \\ ${ }^{1}$ Institute of Pathology, University Medical Center Göttingen, Göttingen, Germany; ${ }^{2}$ Department of Gastroenterology and Gastrointestinal \\ Oncology, University Medical Center Göttingen, Göttingen, Germany \\ Contributions: (I) Conception and design: All authors; (II) Administrative support: All authors; (III) Provision of study materials or patients: None; \\ (IV) Collection and assembly of data: None; (V) Data analysis and interpretation: None; (VI) Manuscript writing: All authors; (VII) Final approval of \\ manuscript: All authors. \\ Correspondence to: Prof. Philipp Ströbel. Institute of Pathology, University Medical Center Göttingen, Robert-Koch-Str. 40, D-37075 Göttingen, \\ Germany. Email: philipp.stroebel@med.uni-goettingen.de.
}

\begin{abstract}
Neuroendocrine tumors of the thymus (tNET) and mediastinum are very rare neoplasms with scarce available data. All subtypes [typical and atypical carcinoid tumors (TC and AC), large cell neuroendocrine and small cell carcinoma (SCC)] observed elsewhere in the body occur also in the mediastinum and show only few if any organ-specific morphological differences. Although all available data suggest that the broad principles that govern the biology (and hence) the classification of these tumors in general apply also to tNET, there are a few noteworthy peculiarities related e.g., to risk factors, relative frequency and also to molecular genetic features. In this review, we will briefly summarize current knowledge on tNET with a special emphasis on shared and private features in comparison e.g., with pulmonary NET, which have traditionally been regarded the next closely related NET group.
\end{abstract}

Keywords: Thymus; neuroendocrine; carcinoid tumor; large cell neuroendocrine carcinoma (LCNEC); small cell carcinoma (SCC); review

Submitted Oct 13, 2016. Accepted for publication Dec 26, 2016.

doi: $10.21037 /$ jtd.2017.02.02

View this article at: http://dx.doi.org/10.21037/jtd.2017.02.02

\section{Introduction}

Neuroendocrine tumors of the thymus (tNET) are very rare, only accounting for about $5 \%$ of all tumors in the thymus and mediastinum, and have an estimated incidence of 1 per 5 million people (1-3). Despite some differences in etiology (e.g., role of cigarette smoking), epidemiology, and genetics, the nomenclature of tNET traditionally followed that of their pulmonary counterparts. Thus, in the most recent fascicle of the World Health Organization (WHO) classification of tumors of the lung, pleura, thymus and heart (4), neuroendocrine tumors of the thymus and lung are separated into typical (low grade) and atypical (intermediate grade) carcinoid tumors, large cell neuroendocrine carcinomas (LCNEC) (5), and small cell carcinomas (SCC) (Table 1). Like in most other organs (6), "low/intermediate grade" typical and atypical carcinoids (TC and AC) on the one hand and "high grade" LCNEC and SCC are believed to be unrelated tumors and not to represent successive stages of de-differentiation, in spite of some overlapping genetic features (7). Defining characteristics of TC and $\mathrm{AC}$ and LCNEC include neuroendocrine morphology on H \& E stainings, "neuroendocrine blood vessels", and expression of neuroendocrine markers. Of note, the diagnosis of SCC is primarily based on $\mathrm{H} \& \mathrm{E}$ morphology and does not require demonstration of neuroendocrine markers by immunohistochemistry. Since the current definition of LCNEC (non-small cell morphology and $>10$ mitoses/10 HPF) includes tumors with just 11 mitoses as well as tumors with $>100$ mitoses, it is likely that this category comprises a (so far undefined) subgroup of "large cell NET" tumors with a lower mitotic rate and an intermediate prognosis, as emerging also in other organ systems.

Occasionally, tNET can occur together with a thymoma or (non-neuroendocrine) thymic carcinoma. Per definition, tumors with a component of a carcinoid tumor (typical/ 
Table 1 Nomenclature and classification of thymic NET

\begin{tabular}{llll}
\hline Low grade & Intermediate grade & High grade & \\
\hline Typical carcinoid: no necrosis; & Atypical carcinoid: necrosis & LCNEC: $>10$ mitosis $/ 2 \mathrm{~mm}^{2}$; no small cell & SCC: small cell morphology; \\
$<$ mitoses $/ 2 \mathrm{~mm}^{2}$ & and/or 2-10 mitoses $/ 2 \mathrm{~mm}^{2}$ & morphology & $>10 \mathrm{mitosis} / 2 \mathrm{~mm}{ }^{2}$ \\
\hline
\end{tabular}

NET, neuroendocrine tumors; LCNEC, large cell neuroendocrine carcinoma; SCC, small cell carcinoma.

atypical) and a thymoma should be reported as e.g., "typical thymic carcinoid (20\%) in combination with a type B2 thymoma (80\%)". In tumors with a combination of thymic carcinoma and a carcinoid tumor, the diagnosis should start with the predominant component and its relative percentage. Tumors containing a component of large cell neuroendocrine carcinoma or SCC are classified as "combined large cell neuroendocrine carcinoma" or "combined SCC" regardless of the size of the neuroendocrine component (4).

tNET do not show organ-specific histological or immunohistochemical features. Accordingly, it is so far not possible in most cases to reliably diagnose a tNET based on morphology alone and clinical information is required to confirm a thymic primary tumor. As for carcinoid tumors of the lung, there is an association between thymic carcinoid tumors and multiple neuroendocrine neoplasia syndrome 1 (MEN-1). In addition to the "standard" organs (parathyroid glands, anterior pituitary, and pancreas/duodenum), MEN1 carriers frequently also develop NETs of the foregut, e.g., bronchial, thymic, and gastric ECLomas (8). About $25 \%$ of TC and AC occur in the context of MEN-1 (9), although the $M E N-1$ gene (Chr. 11q13) is never mutated in these tumors (10), in contrast to some sporadic tNET (11). LCNEC and SCC, in contrast, are not associated with MEN-1. Both patients with pulmonary and thymic NET are at an increased risk to develop other malignancies such as carcinomas of the breast or prostate. In contrast to lung carcinoids, where most cases (70-90\%) belong to the typical carcinoid category and occur predominantly in middle-aged females, the vast majority of cases in the thymus are ACs with a marked male predominance (4). Putative precursor lesions such as the "diffuse idiopathic pulmonary neuroendocrine cell hyperplasia (DIPNECH)" have not been described in the thymus. The genetic profile of thymic carcinoids is clearly different from pulmonary carcinoids $(7,12-17)$. SCCs of the thymus are exceedingly rare, again in contrast to their much more prevalent pulmonary counterparts. Interestingly, the few available molecular studies have so far failed to show genetic differences between pulmonary and bona fide thymic high grade NEC (LCNEC and SCC).

About half of patients with tNET come to clinical attention by local symptoms such as chest pain, cough and respiratory distress. Paraneoplastic syndromes such as Cushing's syndrome or hypercalcemia are mainly associated with TC and AC and are rare in high-grade tumors. Carcinoid syndrome is rare (3). NET have a pronounced tendency for lymph node (18) and distant metastases. Particularly the lungs and bones are commonly affected, while the liver, pancreas and adrenal glands are rarely involved (3). The current histological classification of tNET appears prognostically relevant when central histopathological case review is performed: reported 5 -year survival rates of TC varied from $50-100 \%$ vs. $30-80 \%$ in AC vs. 30-66\% in LCNEC and $0 \%$ in SCC. Other relevant prognostic factors are tumor stage, resection status, and tumor recurrence $(7,8,19,20)$, although neither the Masaoka staging (7), nor the TNM classification as proposed by ITMIG (21) seem to be ideally suited for reliable staging of tNETs.

\section{Typical carcinoid tumors (ICD-0 8240/3)}

TC of the thymus are defined by their unequivocal neuroendocrine morphology in $\mathrm{H}$ \& E stainings (uniform epithelial cells with basophilic cytoplasm and usually bland cytological features with "salt-andpepper" chromatin, delicate angulated blood vessels) and by strong and diffuse expression of neuroendocrine markers on immunohistochemistry. The mitotic rate is per definition $<2$ per $2 \mathrm{~mm}^{2}$ (about $10 \mathrm{HPF}$ in most microscopes). Assessment of proliferation through Ki67 immunohistochemical staining is routinely applied in most laboratories, but has not been formally established so far for the purpose of tNET classification. Necrosis is absent. Approximately $20 \%$ of tNET belong to the TC category (7) with more than two thirds of these cases occurring in middle-aged men (49 years; 31-66 years) (3). About 25\% of cases are associated with MEN-1 (9). There is no 

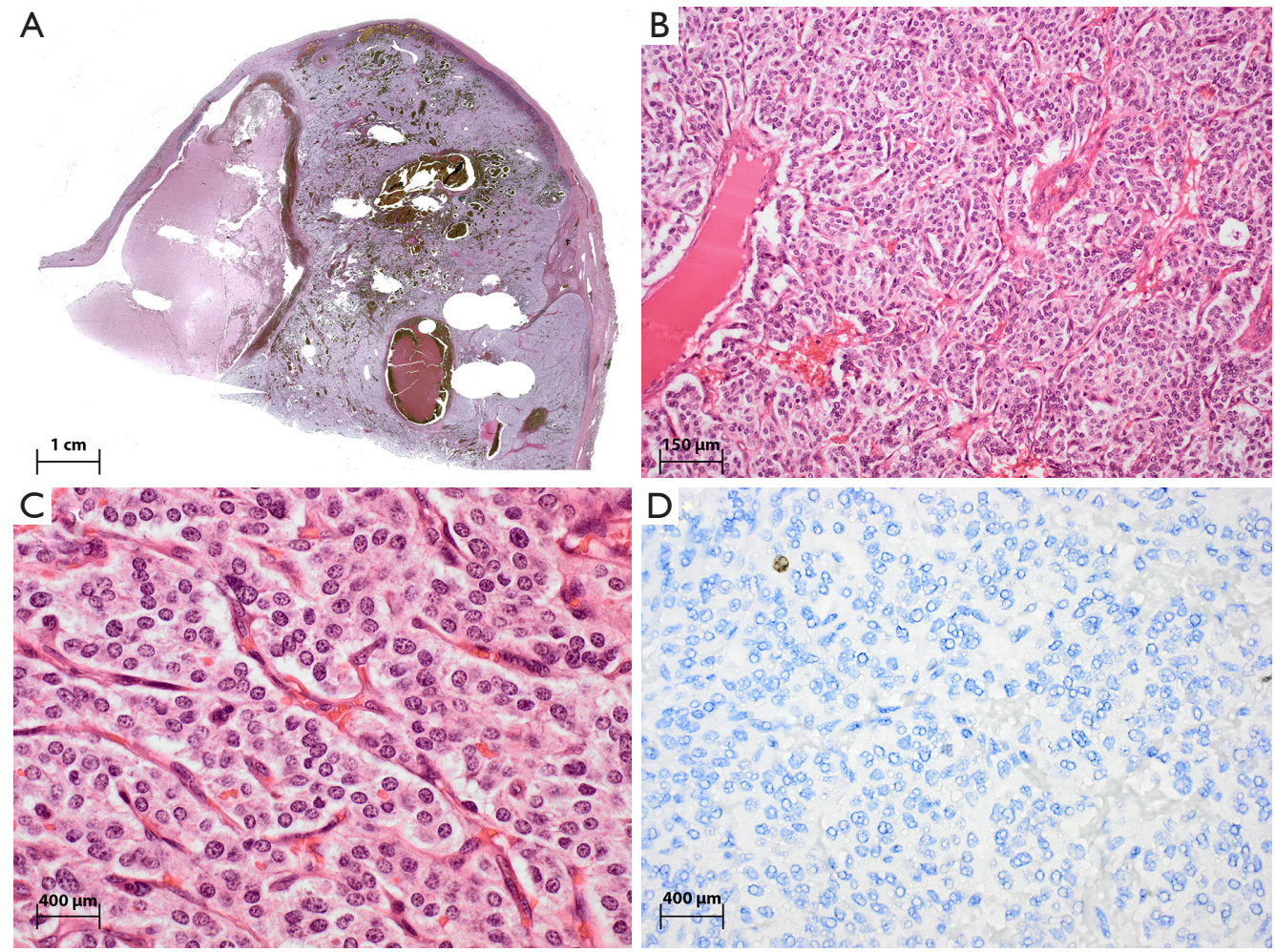

Figure 1 Histomorphology of typical carcinoid tumors of the thymus. (A) Low power view shows a vaguely lobulated mass with cystic change; (B) tumor cells in in this example grow in trabecular structures with some perivascular spaces; (C) the neoplastic cells are uniformly small, round to oval with scant and pale eosinophilic cytoplasm, with relatively small, bland nuclei containing fine granular chromatin. Note the numerous delicate blood vessels that are typical for endocrine tumors; (D) ki67-index is below $1 \%$ in this case. Overall, there are no specific histological features to suggest that this case is of thymic origin and clinical information is essential. (A-C) Hematoxylin \& Eosin; (D) DAB immunohistochemistry on paraffin.

significant age difference between patients with MEN1 associated tumors and patients with sporadic tumors. Virtually all MEN-1-associated thymic NETs occurred in males.

Around half of the patients show local symptoms such as pain, cough, dyspnea or respiratory distress. Up to $30 \%$ of cases are associated with paraneoplastic phenomena due to hormone production of the tumor, including Cushing's syndrome with and without cutaneous hyperpigmentation $(22,23)$, hypercalcemia, and hypophosphatemia through production of parathyroid hormone-like substances or from primary hyperparathyroidism in the context of the MEN1 syndrome (24). Rarely acromegaly (25) or syndromes of inappropriate antidiuretic hormone secretion have been reported (26).

Most tumors are not encapsulated and can be grossly invasive. The size ranges from $2-20 \mathrm{~cm}(2,3,20)$.
Cases associated with Cushing's syndrome tend to be smaller due to earlier detection. Calcifications are more frequently encountered in thymic (30\%) than extrathymic neuroendocrine tumors (2), possibly due to the fact that ACs (with necrosis and subsequent calcifications) are more frequent in the thymus. Histologically, most tumors $(>50 \%)$ show a trabecular growth pattern and rosette formation with nuclear palisading (Figure 1), Additionally, patterns such as ribbons (trabeculae), festoons, solid nests and glandular structures are also common. Lymphovascular invasion is frequent. The tumor cells are uniformly small, round to oval with scant and pale eosinophilic cytoplasm, with relatively small, bland nuclei containing fine granular chromatin. Sometimes larger cells with abundant granular cytoplasm occur. There are numerous carcinoid variants, including spindle cell (27-29), pigmented (5,30), amyloid (27), oncocytic (which can be rarely associated 
with MEN-1 or Cushing's syndrome) (31,32), mucinous $(33,34)$, angiomatoid (35) and sarcomatous variants (with fibrosarcomatous, myoid, osseous or chondroid differentiation) (36,37).

Carcinoids are virtually all immunoreactive for keratins (AE1/3, CAM5.2), often with a characteristic perinuclear accentuation. More than $50 \%$ of tumor cells are strongly positive for neuroendocrine markers such as synaptophysin, chromogranin, and NSE. Specific hormones such as ACTH or somatostatin are often focally positive, but there is no close correlation between immunohistochemical expression and clinical symptoms. TTF1 is usually negative $(38,39)$. The histological differential diagnosis includes paragangliomas (keratin negative) (40), thymomas (especially type A), and medullary thyroid carcinoma. Another important differential diagnosis is pulmonary carcinoid tumor with mediastinal involvement. Thymic NETs have been reported to express Pax8 in about a third of cases, and are usually TTF1 negative (39), while pulmonary NETs frequently have an opposite phenotype. Carcinoids can also occur as so-called somatic malignancies arising in a mediastinal teratoma (41). The mucinous carcinoid variant can resemble metastatic mucinous carcinoma from the gastrointestinal tract or breast.

Most low grade NET are characterized by a low mutation load and few chromosomal aberrations (8), and tNET are no exception to this rule. TC are the subgroup of thymic NET with the fewest genetic alterations. Up to $40 \%$ of cases show no changes detectable by comparative genomic hybridization $(7,42)$. Recurrent alterations include gains of chromosome $1,2 \mathrm{q} 24,7,8 \mathrm{p}, 8 \mathrm{q}, 9 \mathrm{q} 13$, $11 \mathrm{q} 23$ and 22 and loss of chromosome $1 \mathrm{p}, 3 \mathrm{p} 11,6 \mathrm{q}, 10 \mathrm{q}$, and $13 \mathrm{q}(7,42,43)$. The gene locus of the MEN-1 gene on chromosome $11 \mathrm{q} 13$ is usually unaltered $(9-11,42,44)$.

Approximately $50 \%$ of patients develop regional lymph node or distal metastasis. Bone and lung are frequently involved; other rare sites include liver, pancreas, and adrenal glands (3).

In operable cases, surgical resection of the tumor is the therapy of choice. There are no reliable data on the significance and sequence of chemotherapy and radiotherapy. Response to somatostatin analogues alone or in combination e.g., with sunitinib, has been described in some cases (45-47). There is insufficient published data on the prognosis of thymic carcinoids. The available data suggest that the prognosis of typical carcinoids is slightly better than atypical carcinoids (7). The 5 -year survival rate, depending on the study, ranges from $50-100 \%(3,7,8)$ with a median survival of 126 months (7).

\section{Atypical carcinoid tumors (ICD-0 8249/3)}

AC of the thymus differs from typical carcinoid tumors by their slightly increased mitotic rate $(2-10$ per $2 \mathrm{~mm}^{2} / 10 \mathrm{HPF}$ ) and/or focal necrosis. Any area of necrosis, regardless of its size, justifies the diagnosis of AC (Figure 2). Cytological atypia is often slightly more pronounced in AC than in TC. Diffuse (so-called lymphoma-like) areas have been described. The immunohistochemical findings and differential diagnostic considerations in AC are essentially identical with those in TC.

AC account for about $40-50 \%$ of all tNET $(7,48)$. They are mainly found in middle-aged adults (48-55 years), rarely in children and adolescents. Up to $70 \%$ of patients are men. The clinical presentation is identical to TC.

The genetic alterations of AC also overlap with those of TC, but their average mutation frequency is higher. The most commonly chromosomal alterations include gains on chromosome 1q, 5p, 5q, 7p, 7q, 8q, 12q24, 17q and 20q, and losses on chromosome 3p, 3q, 4q, 5q, 6q, 10q, 11q and 13q.

At least a quarter of the tumors have metastasized at the time of diagnosis to mediastinal, cervical or supraclavicular lymph nodes. About half of the tumors are locally advanced (Masaoka tumor stage III) or have metastasized to the lungs, brain, bone, liver or adrenal glands.

Depending on the series, the 5 -year survival of patients with AC is slightly worse than of patients with TC, with a range from $20-80 \%$ depending on the series $(2,7,8)$. Relapses can occur after several years (49).

\section{LCNEC (ICD-0 8013/3)}

LCNEC of the thymus are high-grade tumors with neuroendocrine morphology, immunohistochemical expression of neuroendocrine markers, high mitotic rate (>10 per $2 \mathrm{~mm}^{2} / \mathrm{HPF}$ ), and often extensive necrosis.

The macroscopy of LCNEC is identical to other thymic neuroendocrine tumors. LCNEC is a tumor with non-small cell morphology and a mitotic rate that by definition exceeds 10 per $10 \mathrm{HPF}$, but is often much higher. Necrosis is almost always present and often extensive. Large tumor cells, including anaplastic giant cells, are more common than in atypical carcinoids (50). Architectural features common to neuroendocrine tumors (nesting, trabeculae, rosettes), if present, are often less obvious and less organized than in atypical carcinoids. Due to the definition of LCNEC, 
A

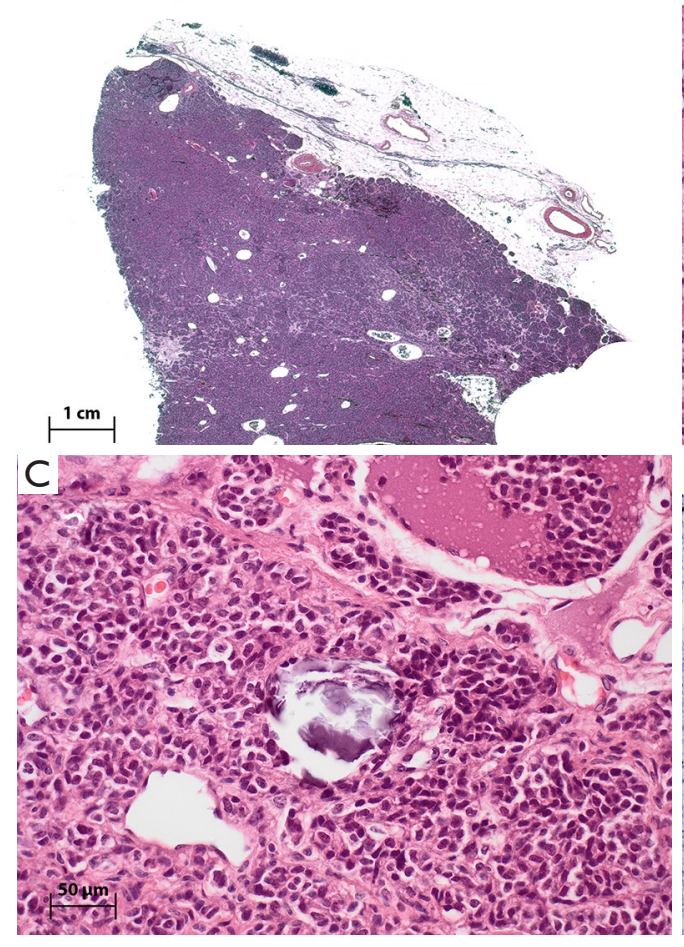

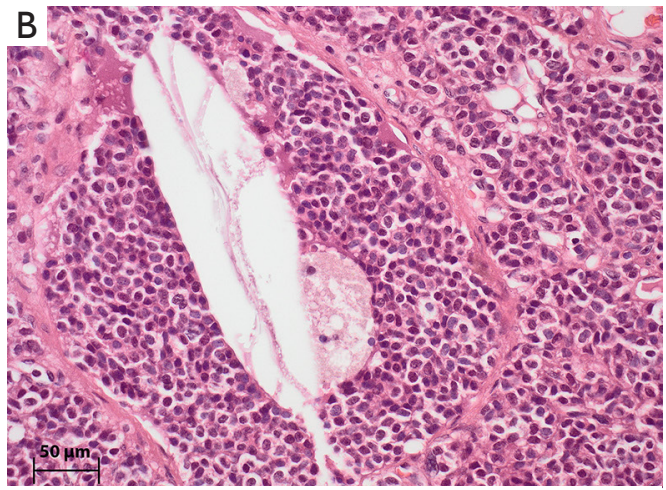

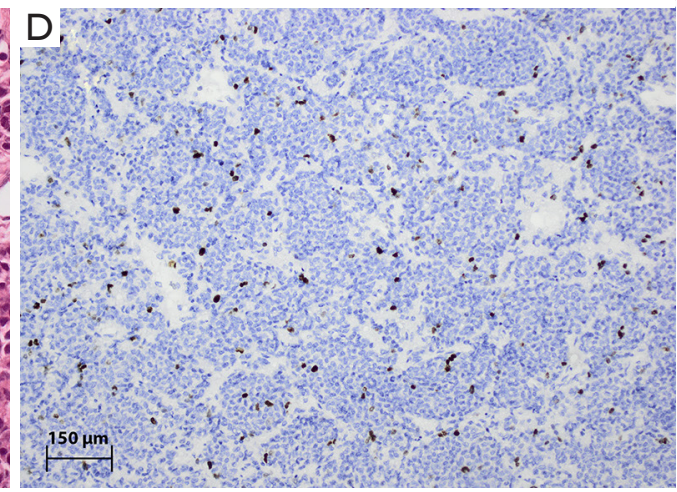

Figure 2 Atypical carcinoid tumor (AC) of the thymus. (A) Low power view showing a solid tumor consisting of small nodules next to an atrophic thymus (upper third of the image); (B) higher magnification shows a small area of tumor necrosis and a cholesterol cleft. Presence of even small foci of unequivocal necrosis places a tumor in the atypical carcinoid category; (C) calcifications are a frequent finding in thymic carcinoid tumors and are thought to be somewhat more frequent than in other organs; (D) this case had 2 mitoses per 10 HPF and a ki67 index of $5 \%$. (A-C) Hematoxylin \& Eosin; (D) DAB immunohistochemistry on paraffin.

which is based on the mitotic rate, the histomorphology of LCNEC is quite variable. In addition to tumors with a "low grade morphology" (i.e., solid or trabecular growth pattern similar to the AC, moderate atypia, but mitotic rate $>10$ per $2 \mathrm{~mm}^{2}$ ), this category includes also tumors with high grade morphology in which the mitotic rate is often higher than 20 per 10 HPF (Figure 3).

LCNEC account for about $15-25 \%$ of neuroendocrine thymic tumors $(51,52)$ and affect twice as many men as women. The estimated incidence is 1 per 20 million inhabitants (1). Most patients are in their middle to late adulthood (median age 51). Approximately half of the patients present with chest pain, dyspnea or upper inflow congestion. The other half of the patients are asymptomatic $(51,52)$. Paraneoplastic phenomena through hormone production (for example Cushing's syndrome) are rare (53).

Most tumors show a strong expression of neuroendocrine markers, such as synaptophysin, chromogranin, or CD56. Keratins (such as AE1/3 or Cam5.2) are always detected and show sometimes a paranuclear accentuation. CD117 is positive in some cases, whilst CD5 is not expressed (50). In cases with expression of TTF1, a pulmonary primary tumor must be excluded through correlation with clinical findings.

About half of thymic carcinomas show a focal and usually rather weak expression of neuroendocrine markers (54). This "aberrant" expression must be distinguished from true neuroendocrine differentiation (suggestive morphology, strong, unique expression of neuroendocrine markers in $>50 \%$ of tumor cells). LCNEC containing a second component of either thymoma or non-neuroendocrine thymic carcinoma are classified as "combined LCNEC", regardless of the relative proportion of the neuroendocrine component.

The molecular changes of LCNEC overlap with those of carcinoid tumors, but the average number of alterations per tumor is higher. Gains on chromosome 1q, 6p, 7, 8q (MYC), $12 \mathrm{q}$ and 14 and losses of chromosome $3,4 \mathrm{q}$ and $13 \mathrm{q}$ are among the most common recurrent changes. Alterations 

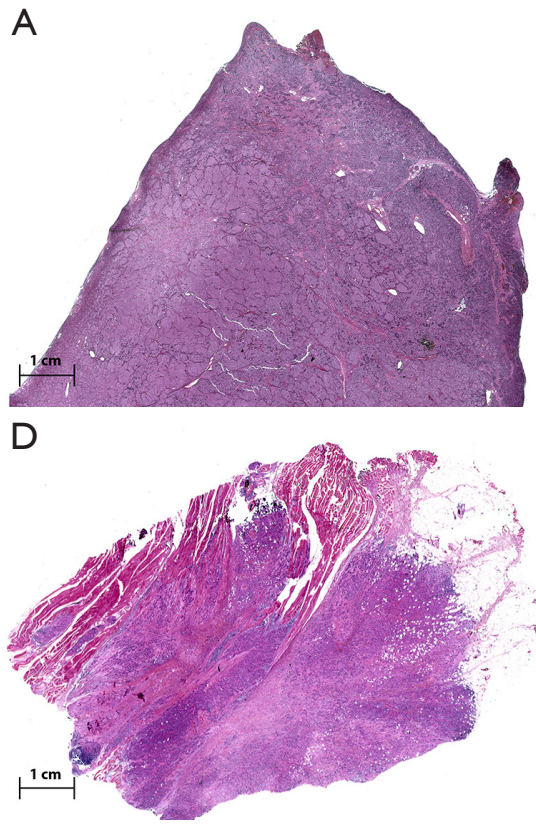
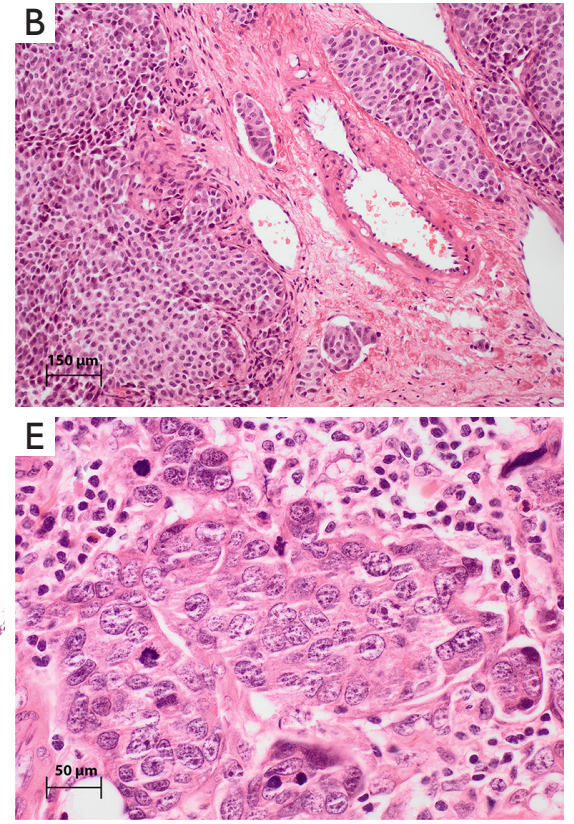
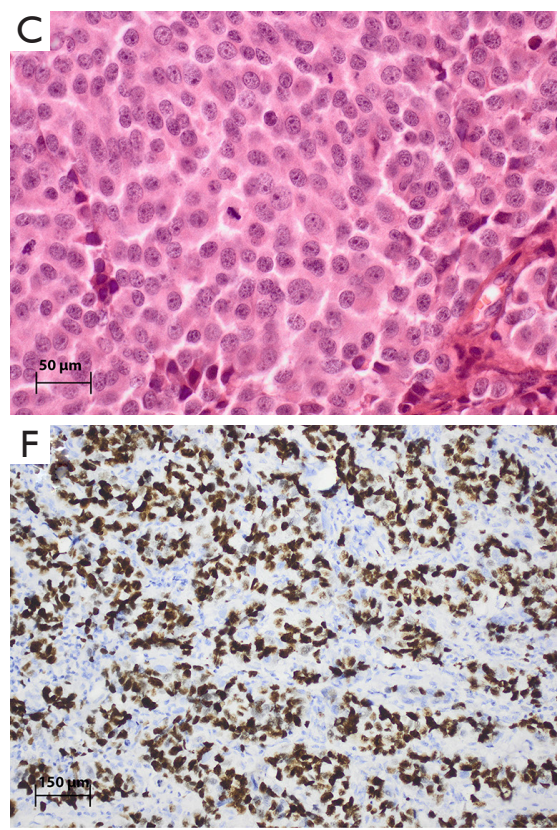

Figure 3 The morphological spectrum of large cell neuroendocrine carcinoma (LCNEC) of the thymus. (A-C) Example of a LCNEC that retained some features of well-differentiated (carcinoid) tumors; (D-F) example of a LCENC with high grade morphology. (A) Necrosis is not evident at this low power magnification; (B) higher magnification shows multiple foci of blood vascular invasion and (C) several mitoses in one visual field; (D) low power magnification shows a widely invasive tumor; (E) higher magnification shows vascular invasion, high grade nuclear morphology, and several mitoses; $(\mathrm{F})$ the ki67 index in this example was >75\%. (A-E) Hematoxylin \& Eosin; (F) DAB immunohistochemistry on paraffin.

found only in LCNEC include gains on $2 p, 9 p$ and $17 q$ and losses on chromosome 4p, 8p, 9p, and 18p (7). These changes do not differ significantly from those in pulmonary LCNEC (7). Additionally, LCNEC do not occur in MEN1 syndrome patients (44,55-58).

At the time of diagnosis, about $75 \%$ of tumors show an infiltration of adjacent organs or distant metastases e.g., to spine and liver $(51,59)$.

The reported 5 -year overall survival ranges from $30-66 \%(7,52)$.

\section{SCC (ICD-0 8041/3)}

SCC of the thymus are high-grade tumors with small cell morphology (scant cytoplasm, often fusiform tumor cells with ill-defined cell membranes, and nuclei with "pepperand salt-chromatin" without prominent nucleoli). Crush artefacts, high mitotic count and extensive necrosis are characteristic findings.

The histomorphology of thymic SCC is identical to SCC elsewhere in the body. Tumor cells are small (usually
$<3$ times the diameter of a small resting lymphocyte) and the cytoplasm is very scant. The mitotic activity is usually very high (over 50 per $2 \mathrm{~mm}^{2}$ ). The tumor cells are vulnerable and therefore often show crush artefacts. Tumor cells also display a high nuclear to cytoplasmic ratio, scant cytoplasm, nuclear molding, and finely granular nuclear chromatin. Nucleoli are inconspicuous or absent. Apoptotic bodies are often numerous (Figure 4). When SCC present with a second component of thymoma or non-endocrine thymic carcinoma, the tumor is by definition classified as "combined SCC", regardless of the relative size of the neuroendocrine component.

SCC accounts for only about $10 \%$ of all tNET with an estimated incidence of 1 per 50 million inhabitants (1). It affects older men and women (median age 58). Unlike the other subtypes, it is not more prevalent in men than in women.

Most but not all tumors show expression of keratins. Expression of neuroendocrine markers is frequent, but not required for the diagnosis. In analogy to SCC in other (extrapulmonary) organs, the detection of TTF1 does 

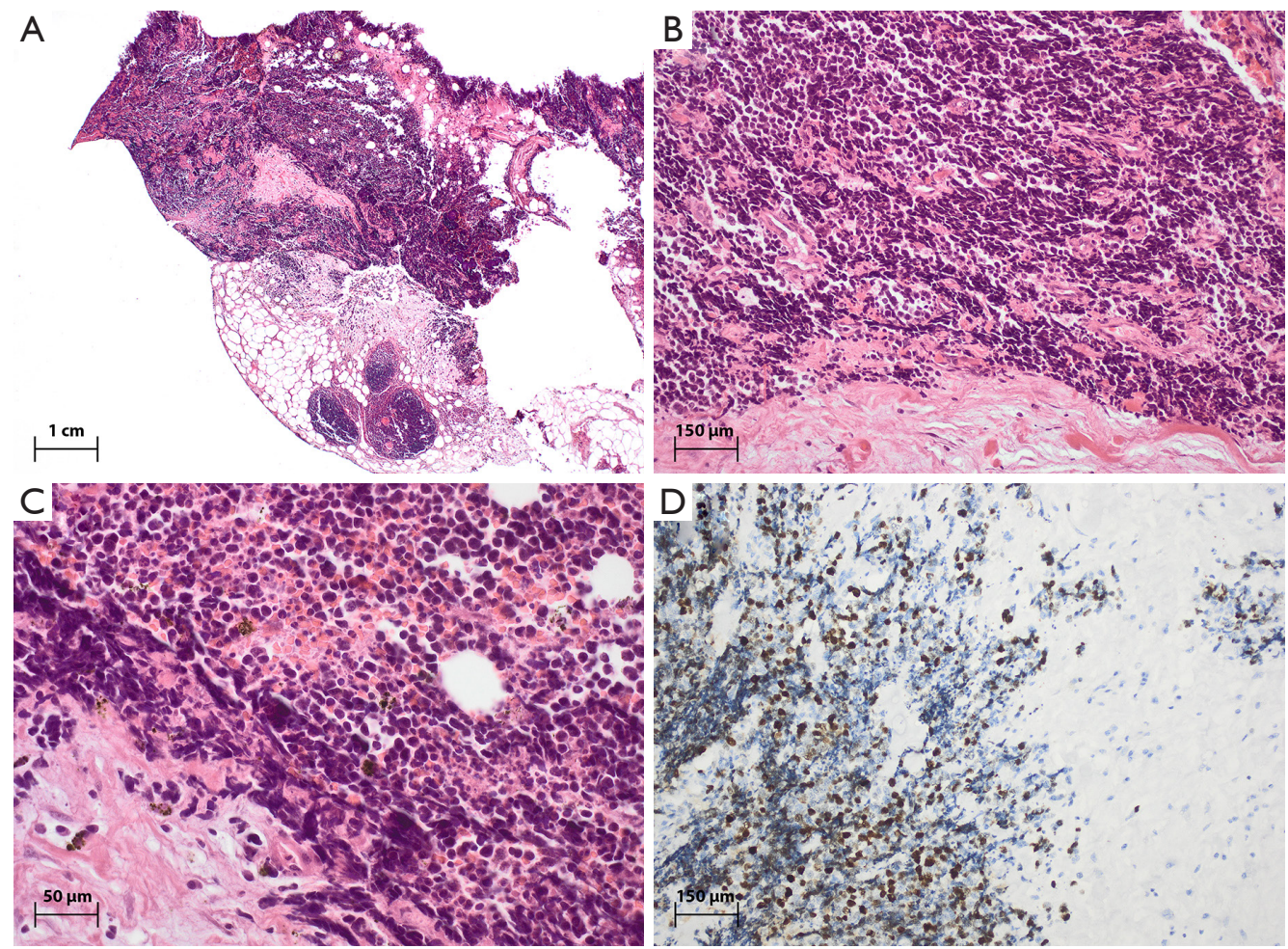

Figure 4 Morphology of small cell carcinoma (SCC) of the thymus. (A) Low power view shows a widely infiltrative tumor next to a thymic remnant (lower half of the image); $(\mathrm{B}, \mathrm{C})$ higher magnification shows the typical small cell morphology with crush artifacts of the vulnerable tumor cell nuclei as well as numerous apoptosis and small foci of necrosis; (D) due to the marked nuclear artefacts, the ki67 index is sometimes difficult to assess, but is usually $>70 \%$. This case stained positive for neuroendocrine markers (not shown), although neuroendocrine differentiation is not required for the diagnosis, if the morphology and proliferation rate are adequate. (A-C) Hematoxylin \& Eosin; (D) DAB immunohistochemistry on paraffin.

not prove a primary tumor of the lung, but should always prompt close correlation with clinical findings. In addition, the differential diagnosis includes other small cell neoplasms such as T-lymphoblastic lymphoma or small cell sarcomas (such as the PNET/Ewing tumor group), which must be excluded by appropriate immunohistochemical or molecular studies.

The very sparse published data on SCC show multiple chromosomal aberrations partially overlapping with those of carcinoid tumors and LCNEC. The genetic profile of thymic SCC does not differ significantly from pulmonary SCC (7). SCC do not occur in MEN-1 syndrome patients $(44,57,58,60,61)$.

At the time of diagnosis, most tumors are in an advanced stage with infiltration of neighboring organs, such as lungs or pericardium, or with distant metastases to the lung, liver, bone or brain $(7,55)$. Symptoms include weight loss, sweating, chest pain, cough, and vena cava superior syndrome $(55,62,63)$.

Prognosis is poor with a 5 -year survival rate of $0 \%$ (64). Median survival is 13.75 months (range, 13-26 months) $(7,55,62,63)$.

\section{Conclusion and future developments}

Thymic neuroendocrine tumors are very rare epithelial neoplasms. Although clinical and molecular data are sparse, their general behaviour and genetic makeup appears to be very similar to corresponding tumors elsewhere in the body. Based on these observations, it is very likely that the classification of tNET will continue to align with the emerging concepts in other organ systems. However, many questions relating to patient management and therapy remain open and more studies on these tumors are required to elucidate immunohistochemical, molecular and genetic peculiarities e.g., in comparison with their much more 
common pulmonary counterparts. Given the rarity of tNET, these studies will likely require a joint international effort.

\section{Acknowledgements}

None.

\section{Footnote}

Conflicts of Interest: The authors have no conflicts of interest to declare.

\section{References}

1. Gaur P, Leary C, Yao JC. Thymic neuroendocrine tumors: a SEER database analysis of 160 patients. Ann Surg 2010;251:1117-21.

2. Moran CA, Suster S. Neuroendocrine carcinomas (carcinoid tumor) of the thymus. A clinicopathologic analysis of 80 cases. Am J Clin Pathol 2000;114:100-10.

3. Soga J, Yakuwa Y, Osaka M. Evaluation of 342 cases of mediastinal/thymic carcinoids collected from literature: a comparative study between typical carcinoids and atypical varieties. Ann Thorac Cardiovasc Surg 1999;5:285-92.

4. Ströbel P, Marx A, Chan JK, et al. Thymic neuroendocrine tumours. In: Travis WD, Brambilla E, Burke AP, et al. editors. WHO classification of tumours of the lung, pleura, thymus and heart. 4th edition. Lyon: International Agency for Research on Cancer press, 2015:234-41.

5. Lagrange W, Dahm HH, Karstens J, et al. Melanocytic neuroendocrine carcinoma of the thymus. Cancer 1987;59:484-8.

6. Fernandez-Cuesta L, Peifer M, Lu X, et al. Frequent mutations in chromatin-remodelling genes in pulmonary carcinoids. Nat Commun 2014;5:3518.

7. Ströbel P, Zettl A, Shilo K, et al. Tumor genetics and survival of thymic neuroendocrine neoplasms: a multiinstitutional clinicopathologic study. Genes Chromosomes Cancer 2014;53:738-49.

8. Crona J, Björklund P, Welin S, et al. Treatment, prognostic markers and survival in thymic neuroendocrine tumours. a study from a single tertiary referral centre. Lung Cancer 2013;79:289-93.

9. Teh BT, Zedenius J, Kytölä S, et al. Thymic carcinoids in multiple endocrine neoplasia type 1. Ann Surg 1998;228:99-105.

10. Teh BT, Hayward NK, Walters MK, et al. Genetic studies of thymic carcinoids in multiple endocrine neoplasia type 1 .
J Med Genet 1994;31:261-2.

11. Fujii T, Kawai T, Saito K, et al. MEN1 gene mutations in sporadic neuroendocrine tumors of foregut derivation. Pathol Int 1999;49:968-73.

12. Walch AK, Zitzelsberger HF, Aubele MM, et al. Typical and atypical carcinoid tumors of the lung are characterized by $11 \mathrm{q}$ deletions as detected by comparative genomic hybridization. Am J Pathol 1998;153:1089-98.

13. Ullmann R, Schwendel A, Klemen H, et al. Unbalanced chromosomal aberrations in neuroendocrine lung tumors as detected by comparative genomic hybridization. Hum Pathol 1998;29:1145-9.

14. Zhao J, de Krijger RR, Meier D, et al. Genomic alterations in well-differentiated gastrointestinal and bronchial neuroendocrine tumors (carcinoids): marked differences indicating diversity in molecular pathogenesis. Am J Pathol 2000;157:1431-8.

15. Petzmann S, Ullmann R, Klemen H, et al. Loss of heterozygosity on chromosome arm $11 \mathrm{q}$ in lung carcinoids. Hum Pathol 2001;32:333-8.

16. Ullmann R, Petzmann S, Klemen H, et al. The position of pulmonary carcinoids within the spectrum of neuroendocrine tumors of the lung and other tissues. Genes Chromosomes Cancer 2002;34:78-85.

17. Johnen G, Krismann M, Jaworska M, et al. CGH findings in neuroendocrine tumours of the lung. Pathologe 2003;24:303-7.

18. Kondo K, Monden Y. Lymphogenous and hematogenous metastasis of thymic epithelial tumors. Ann Thorac Surg 2003;76:1859-64; discussion 1864-5.

19. Economopoulos GC, Lewis JW Jr, Lee MW, et al. Carcinoid tumors of the thymus. Ann Thorac Surg 1990;50:58-61.

20. de Montpréville VT, Macchiarini P, Dulmet E. Thymic neuroendocrine carcinoma (carcinoid): a clinicopathologic study of fourteen cases. J Thorac Cardiovasc Surg 1996;111:134-41.

21. Zhao Y, Shi J, Fan L, et al. Evaluation of the proposed International Association for the Study of Lung Cancer (IASLC)/International Thymic Malignancies Interest Group (ITMIG) staging revisions in thymic welldifferentiated neuroendocrine carcinoma patients. Eur J Cardiothorac Surg 2016;49:569-73.

22. de Perrot M, Spiliopoulos A, Fischer S, et al. Neuroendocrine carcinoma (carcinoid) of the thymus associated with Cushing's syndrome. Ann Thorac Surg 2002;73:675-81.

23. Gartner LA, Voorhess ML. Adrenocorticotropic hormone- 
-producing thymic carcinoid in a teenager. Cancer 1993;71:106-11.

24. Yoshikawa T, Noguchi Y, Matsukawa H, et al. Thymus carcinoid producing parathyroid hormone (PTH)-related protein: report of a case. Surg Today 1994;24:544-7.

25. Jansson JO, Svensson J, Bengtsson BA, et al. Acromegaly and Cushing's syndrome due to ectopic production of GHRH and ACTH by a thymic carcinoid tumour: in vitro responses to GHRH and GHRP-6. Clin Endocrinol (Oxf) 1998;48:243-50.

26. Okada S, Ohshima K, Mori M. The Cushing syndrome induced by atrial natriuretic peptide-producing thymic carcinoid. Ann Intern Med 1994;121:75-6.

27. Levine GD, Rosai J. A spindle cell varient of thymic carcinoid tumor. A clinical, histologic, and fine structural study with emphasis on its distinction from spindle cell thymoma. Arch Pathol Lab Med 1976;100:293-300.

28. Wick MR, Rosai J. Neuroendocrine neoplasms of the mediastinum. Semin Diagn Pathol 1991;8:35-51.

29. Moran CA, Suster S. Spindle-cell neuroendocrine carcinomas of the thymus (spindle-cell thymic carcinoid): a clinicopathologic and immunohistochemical study of seven cases. Mod Pathol 1999;12:587-91.

30. Klemm KM, Moran CA, Suster S. Pigmented thymic carcinoids: a clinicopathological and immunohistochemical study of two cases. Mod Pathol 1999;12:946-8.

31. Moran CA, Suster S. Primary neuroendocrine carcinoma (thymic carcinoid) of the thymus with prominent oncocytic features: a clinicopathologic study of 22 cases. Mod Pathol 2000;13:489-94.

32. Yamaji I, Iimura O, Mito T, et al. An ectopic, ACTH producing, oncocytic carcinoid tumor of the thymus: report of a case. Jpn J Med 1984;23:62-6.

33. Ohchi T, Tanaka H, Shibuya Y, et al. Thymic carcinoid with mucinous stroma: a case report. Respir Med 1998;92:880-2.

34. Suster S, Moran CA. Thymic carcinoid with prominent mucinous stroma. Report of a distinctive morphologic variant of thymic neuroendocrine neoplasm. Am J Surg Pathol 1995;19:1277-85.

35. Moran CA, Suster S. Angiomatoid neuroendocrine carcinoma of the thymus: report of a distinctive morphological variant of neuroendocrine tumor of the thymus resembling a vascular neoplasm. Hum Pathol 1999;30:635-9.

36. Kuo TT. Carcinoid tumor of the thymus with divergent sarcomatoid differentiation: report of a case with histogenetic consideration. Hum Pathol 1994;25:319-23.
37. Paties C, Zangrandi A, Vassallo G, et al. Multidirectional carcinoma of the thymus with neuroendocrine and sarcomatoid components and carcinoid syndrome. Pathol Res Pract 1991;187:170-7.

38. Oliveira AM, Tazelaar HD, Myers JL, et al. Thyroid transcription factor-1 distinguishes metastatic pulmonary from well-differentiated neuroendocrine tumors of other sites. Am J Surg Pathol 2001;25:815-9.

39. Weissferdt A, Tang X, Wistuba II, et al. Comparative immunohistochemical analysis of pulmonary and thymic neuroendocrine carcinomas using PAX8 and TTF-1. Mod Pathol 2013;26:1554-60.

40. Weissferdt A, Kalhor N, Liu H, et al. Thymic neuroendocrine tumors (paraganglioma and carcinoid tumors): a comparative immunohistochemical study of 46 cases. Hum Pathol 2014;45:2463-70.

41. Schaefer IM, Zardo P, Freermann S, et al. Neuroendocrine carcinoma in a mediastinal teratoma as a rare variant of somatic-type malignancy. Virchows Arch 2013;463:731-5.

42. Pan CC, Jong YJ, Chen YJ. Comparative genomic hybridization analysis of thymic neuroendocrine tumors. Mod Pathol 2005;18:358-64.

43. Rieker RJ, Aulmann S, Penzel R, et al. Chromosomal imbalances in sporadic neuroendocrine tumours of the thymus. Cancer Lett 2005;223:169-74.

44. Gibril F, Chen YJ, Schrump DS, et al. Prospective study of thymic carcinoids in patients with multiple endocrine neoplasia type 1. J Clin Endocrinol Metab 2003;88:1066-81.

45. Okabe N, Inoue $T$, Watanabe $Y$, et al. Two cases of thymic carcinoid treated with octreotide long-acting repeatable. Gan To Kagaku Ryoho 2014;41:879-83.

46. Dham A, Truskinovsky AM, Dudek AZ. Thymic carcinoid responds to neoadjuvant therapy with sunitinib and octreotide: a case report. J Thorac Oncol 2008;3:94-7.

47. Kulke MH, Lenz HJ, Meropol NJ, et al. Activity of sunitinib in patients with advanced neuroendocrine tumors. J Clin Oncol 2008;26:3403-10.

48. Filosso PL, Yao X, Ahmad U, et al. Outcome of primary neuroendocrine tumors of the thymus: a joint analysis of the International Thymic Malignancy Interest Group and the European Society of Thoracic Surgeons databases. J Thorac Cardiovasc Surg 2015;149:103-9.e2.

49. Tsuchida M, Yamato Y, Hashimoto T, et al. Recurrent thymic carcinoid tumor in the pleural cavity. 2 cases of long-term survivors. Jpn J Thorac Cardiovasc Surg 2001;49:666-8.

50. Chetty R, Batitang S, Govender D. Large cell neuroendocrine carcinoma of the thymus. Histopathology 
1997;31:274-6.

51. Ahn S, Lee JJ, Ha SY, et al. Clinicopathological analysis of 21 thymic neuroendocrine tumors. Korean J Pathol 2012;46:221-5.

52. Shoji T, Fushimi H, Takeda S, et al. Thymic large-cell neuroendocrine carcinoma: a disease neglected in the ESMO guideline? Ann Oncol 2011;22:2535.

53. Saito T, Kimoto M, Nakai S, et al. Ectopic ACTH syndrome associated with large cell neuroendocrine carcinoma of the thymus. Intern Med 2011;50:1471-5.

54. Lauriola L, Erlandson RA, Rosai J. Neuroendocrine differentiation is a common feature of thymic carcinoma. Am J Surg Pathol 1998;22:1059-66.

55. Tiffet O, Nicholson AG, Ladas G, et al. A clinicopathologic study of 12 neuroendocrine tumors arising in the thymus. Chest 2003;124:141-6.

56. Öberg K, Hellman P, Ferolla P, et al. Neuroendocrine bronchial and thymic tumors: ESMO Clinical Practice Guidelines for diagnosis, treatment and follow-up. Ann Oncol 2012;23 Suppl 7:vii120-3.

57. Goudet P, Murat A, Cardot-Bauters C, et al. Thymic neuroendocrine tumors in multiple endocrine neoplasia type 1: a comparative study on 21 cases among a series of 761 MEN1 from the GTE (Groupe des Tumeurs Endocrines). World J Surg 2009;33:1197-207.

Cite this article as: Bohnenberger H, Dinter H, König A, Ströbel P. Neuroendocrine tumors of the thymus and mediastinum. J Thorac Dis 2017;9(Suppl 15):S1448-S1457. doi: doi: $10.21037 /$ jtd.2017.02.02
58. Sakurai A, Imai T, Kikumori T, et al. Thymic neuroendocrine tumour in multiple endocrine neoplasia type 1: female patients are not rare exceptions. Clin Endocrinol (Oxf) 2013; 78:248-54.

59. Dutta R, Kumar A, Julka PK, et al. Thymic neuroendocrine tumour (carcinoid): clinicopathological features of four patients with different presentation. Interact Cardiovasc Thorac Surg 2010;11:732-6.

60. Teh BT. Thymic carcinoids in multiple endocrine neoplasia type 1. J Intern Med 1998;243:501-4.

61. Ferolla P, Falchetti A, Filosso P, et al. Thymic neuroendocrine carcinoma (carcinoid) in multiple endocrine neoplasia type 1 syndrome: the Italian series. J Clin Endocrinol Metab 2005;90:2603-9.

62. Wick MR, Scheithauer BW, Weiland LH, et al. Primary thymic carcinomas. Am J Surg Pathol 1982;6:613-30.

63. Kuo TT, Chang JP, Lin FJ, et al. Thymic carcinomas: histopathological varieties and immunohistochemical study. Am J Surg Pathol 1990;14:24-34.

64. Moran CA, Suster S. Thymic neuroendocrine carcinomas with combined features ranging from well-differentiated (carcinoid) to small cell carcinoma. A clinicopathologic and immunohistochemical study of 11 cases. Am J Clin Pathol 2000;113:345-50. 